\title{
Occurance of Dizziness in patients with Tinnitus Complaint
}

\begin{abstract}
Amanda Câmara Miranda ${ }^{1}$, Thaís Mendonça Maia Wanderley Cruz de Freitas ${ }^{2}$, Emanuelle Sintya Santos Santana do Nascimento ${ }^{3}$, Claúdia Carneiro da Silva ${ }^{1}$, Karla Cybelle Bezerra Cavalcanti Alcoforado ${ }^{4}$, Karlin Fabianne Klagenberg ${ }^{5}$ and Marine Raquel Diniz da Rosa ${ }^{6}$
\end{abstract}

${ }^{1}$ Master student in Cognitive Neuroscience and Behaviour Universidade Federal da Paraíba, Brazil

${ }^{2}$ Master student in Speech Therapy, Universidade Federal da Paraíba, Brazil

${ }^{3}$ Master in Model and Decisions, Universidade Federal da Paraíba, Brazil

${ }^{4}$ Lecturer in Centro Universitário de João Pessoa, Brazil

${ }^{5}$ Doctor in Communication Disorders through the Universidade Tuiuti do Paraná, Brazil

${ }^{6}$ Adjunct Lecturer of the Speech therapy course of the Universidade Federal da Paraíba, Brazil

*Corresponding author: Marine Raquel Diniz da Rosa, Adjunct Lecturer of the Speech therapy course of the Universidade Federal da Paraíba, Brazil

\begin{abstract}
Introduction: Dizziness is a regular complaint, often accompanied by other symptoms, especially tinnitus. Tinnitus is one of the three major otoneurological manifestations, alongside neurosensorial hearing loss and dizziness, being it, most of the times, the main complaint among patients. The relationship between the vestibular and cochlear system it's rather known, many pathologies can originate from one of both systems.

Objective: Investigate the correlation between the symptoms of tinnitus and dizziness, analyzing the level of disturbance, the sensation of frequency (pitch) and intensity (loudness) of the tinnitus with dizziness complaint.

Methodology: A descriptive, observational and quantifying field study took place. 126 individuals with tinnitus complaint, from both sexes, were studied. The anamnesis was performed approaching audiological symptoms, the THI questionnaire was applied, as well as acuphenometry.

Results: 71 individuals (56,3\%) referred to dizziness complaints associated with the tinnitus; women represented a larger number $(41,3 \%)(p=0,017)$. In regards of the level of disturbance of the tinnitus, most of the patients $18,3 \%$ presented a low level, as for patients without dizziness $14,3 \%$ the quick level was present; the average Pitch is around $4.000 \mathrm{~Hz}$ in both groups, Loudness, on the other hand, was of $22 \mathrm{dBNS}$ for individuals with dizziness and $26 \mathrm{dBNS}$ for individuals without dizziness complaints.

Conclusion: Meaningful results, regarding the relationship between tinnitus and dizziness, were not observed, therefore, it's necessary to investigate if the tinnitus is from vestibular origin in order to seek improvements to the dizziness and thereafter, the tinnitus.
\end{abstract}

Keywords: Dizziness; Tinnitus; Audiology; Speech Therapy

\section{Introduction}

The corporal balance relies on the integrity of the vestibular system (labyrinth, nerve vestibulocochlear, cores, paths and interrelations of the central nervous system), somatosensorial system (receptors, sensors located on tendons, muscles and articulations) and vision [1]. The labyrinth is responsible for the balance and position of the body in location. Dizziness and/or imbalance come 
to be when there is interference, both central and peripheral, in the regular operation of the body balance system. [2]. Dizziness can be defined, according to the Hearing and Balance Committee of the American Academy of Comitê de Audição e Equilíbrio da Academia Americana de Otolaryngology and Head/Neck Surgery [3], as every and any illusory feeling of motion without any real motion in relation to gravity. In the practice clinic, it's one of the most frequent complaints. It affects between $20 \%$ and $30 \%$ of the general population, considering the epidemiological study of Neuhauser and collaborators [4]. In Brazil, an epidemiological study, performed in the city of São Paulo, showed that $42 \%$ of the individuals presented dizziness [5]. Occurrences can be found among any age range, from the first months of birth to the elderly population [6]. Causes can be many, such as: benign paroxysmal postural vertigo (VPPB), vestibular neuritis, Ménière's disease, peristaltic fistula, circulatory, metabolic, hormonal or immunological, cervical spine changes, head trauma and psychoactive disorders, are one of the most common. This way, the symptoms coming from the dizziness may or may not originate from the vestibular system and comprises sensations described in many ways: vertigo (rotatory dizziness), imbalance, fluctuation or instability, presyncope our lipothymia, kinesis (motion sickness), oscillopsia, falling [7]. Dizziness of nonvestibular origin are often ill-defined, most of the times they are labeled as uneasiness, light-headedness, sensation of fainting. Also, in rare cases, they might represent symptoms of the central nervous system and/or may be associated with exclusively ocular disorders, ischemic episodes, metabolic disorder, neurological, cardiac or cervical diseases [8]. However, dizziness is usually due to primary or secondary functional disorders of the vestibular system, it might be classified as rotatory (vertigo - when the illusion of motion has rotatory characteristics) or non-rotatory (when the illusion of motion has no rotatory characteristics). Regular vertigo is more common among peripheral syndromes rather than central ones. The peripheral vertigo is usually aggravated by eye shutting, unlike what happens with central vertigo. Both peripheral and central vertigo can be unleashed or worsen with head motion (this being the most common kind of rotatory dizziness). On the other hand, non-rotatory dizziness may be oscillating, hesitant, fluctuance, wavering, among others. In order to determine the vestibular source an examination of the alterations of the vestibular system is required [9]. Dizziness is usually followed by other symptoms; it's intensity can cause loss of balance and falls. Normally, the dizziness appearance is accompanied by neurovegetative symptoms, megrims, eyesight darkening and lack of concentration.

Dizziness is also highly associated with auditory symptoms, such as hearing loss, sensation of auricular plenitude and, mainly, tinnitus $[10,11]$. The tinnitus is one of the 3 major otoneurological manifestations, alongside neurosensorial hearing loss and dizziness, being it, most of the times, the main complaint among patients, especially elder ones $[12,13]$. The tinnitus, also known as tinnitus, can be defined as auditory illusion, in other words, an endogenous sound illusion, not related to any outside source of stimulation [14]. The presence of tinnitus might be a factor of great negative repercussion in one's life, jeopardizing sleep, concentration during day-to-day and professional, as well as social life. Many times, it affects the emotional balance of the patient, unleashing or worsening states of depression and anxiety [15]. A study performed in the city of são Paulo shows that 22\% (430 individuals) present tinnitus [16]. The tinnitus is as symptom that can be caused by a number of medical conditions: otological affections, neurological, cardiovascular, metabolic, pharmacologic, odontological, psychologic, side effects of medications and possible drug ingestion, such as caffeine, nicotine and alcohol [17]. Up to date theories to explain the source of tinnitus defend the hypothesis that it occurs due to anomalous and spontaneous neural activity in the central pathways, auditory or not, being a consequence of sensory deprivation, aftermath of cochlear lesion $[18,19]$. The description of the tinnitus's characteristics might vary from patient to patient, from "pure tone" sound to a "whistle", a "noise" or even a "whisper", etc. Perceivable in one or both ears, and yet in the head, with no specific side. It can be constant or intermittent, being absent for some periods of time. Its intensity may vary from light to very intense [18]. Patients with dizziness, resistant to various treatments, can be as hard to conduct as patients with high level of tillindus disturbance, these that might happen simultaneously or independently. Both dizziness and noise are extremely common symptoms at the practice clinic, as shown by the study performed by Moreira and collaborators [20] where out of 27 individuals affected by dizziness, 16 (59,2\%) also complained about noise.

The relationship between the vestibular and cochlear systems is rather known. Many pathologies may originate in one of those systems or simultaneously, as well as having one of them as primary source due to influence in other systems functionality. Therefore, it's possible that changes in the posterior labyrinth (Semicircular Canal) fluids may cause tinnitus [21]. It's noticeable that both disorders jeopardize the individual's quality of life. The tinnitus may affect one's sleep, concentration, emotional balance and social life. On the other hand, dizziness, apart from other mentioned symptoms, might hinder the individual's performance during activities that require quick head motions and, also, tasks that imply flexing the torso and the head [22]. Innumerable reports of dizziness among patients with tinnitus complaints were observed in a Multidisciplinary service of attendance to patients with tinnitus. The aforementioned research main goal was to investigate the correlation between dizziness and tinnitus symptoms on those patients, analyzing the level of disturbance, the sensation of frequency (pitch) and intensity (loudness) of tinnitus with dizziness complaint.

\section{Material and Methods}

The present study was performed in one School Clinic of the Speech Therapy Course located in João Pessoa. 126 individuals with tinnitus complaint were evaluated, 81 females and 45 males, ages ranging from 17 to 83 years old, all attended in the Multidisciplinary Tinnitus Service. It was a descriptive research. As for technical procedures, a field study is more fitting, as it tries to deepen between the dizziness and its relationship towards tinnitus. In order to validate these hypotheses, a transversal and observational study, of quantitative characteristics, was performed. In accordance with the 466/12 Resolution of the National Health 
Committee, referring to ethics regarding research that involves human subjects, the study was approved by the Ethics in Research with Human Subjects Committee (prot. N. 0129/12). Clearance was obtained through the signing of the Term of Free and EnlightenedTCL by volunteers and/or responsible for the project. All patients were subjected to the following procedures:

I. Answering anamnesis questions which emphasize the patient's auditory symptoms, in order to collect personal data of the individual; data about the tillindus - location of the tinnitus (right, left, in the head or undetermined), time of onset, how it came to be (gradual, sudden, after noise exposure, other), kind (continuous, pulsatile, intermittent), characteristics (whistle, rain, noise, waterfall, bee, other); and other possible associated symptoms, such as dizziness.

II. In order to obtain more directed information regarding the patient's tinnitus, the Tinnitus Handicap Inventory (THI) questionnaire was applied, as an interview. The THI is a questionnaire that evaluates the severity of the tinnitus, with 25 questions approaching three dimensions: nine questions related to emotional aspects (frustration, anger, irritability, anxiety, depression and insecurity); eleven questions related to functional aspects (stress, concentration, sleep, workplace interference, house responsibilities and social activities); antivideos socials); and five questions related to the catastrophic aspects (despair, lack of self-control, inability of acceptance, perception of terrible illness) (Lim et al, 2010). Those 25 questions allow the following possibilities of answer: "yes", "no" and "sometimes", each having a score of " 4 points", "0 points" and " 2 points", respectively. This said, each question will add points to its category, be it functional, emotional or catastrophic, and the total sum, varying from 0 to a 100. Depending on the result, the level of disturbance caused by the tinnitus might be classified as, by the total sum, as:

a) LEVEL 1 (Quick): Score 0 - 16. Only perceived in quiet environments.

b) LEVEL 2 (Light): Score 18 - 36. Easily masked by environmental and easily forgotten during day-to-day activities.

c) LEVEL 3 (Moderated): Score 38 - 56. Perceived in the presence of background noise, however, day-to-day activities can still be performed.

d) LEVEL 4 (Severe): Score 58 - 76. Almost always perceived, leads to disturbance in sleep patterns and may interfere in daily activities.

e) LEVEL 5 (Catastrófico): Score 78 - 100. Always perceived, sleep patterns disturbances, difficulty performing daily activities.

Later on, acuphenometry was performed in order to obtain data regarding the sensation of intensity (loudness) and frequency (pitch) of the tinnitus. The test took place in an acoustic cabin, using the audiometer model AVS 500, of the Vibrasom brand. To unilateral tinnitus, the sound was supplied to the contralateral ear and, if bilateral, to the ear with best hearing [23]. In order to identify what kind of tinnitus, the Pure Continuous Tone, Pulsatile Pure Tone and Modulated Frequency were presented, in audible intensity, so that the patient could choose which resembles his own tinnitus more. To measure Pitch, the chosen tone was presented in frequencies ranging from 125 to $8000 \mathrm{~Hz}$. And to investigate loudness, the same tone was presented in the frequency identified by the patient, with audible intensity, incrementing $1 \mathrm{db}$.

\section{Data Analysis}

The data was registered in an Excel spreadsheet for further analysis. Initially, a static descriptive analysis was performed, in order to verify the frequency of the variables studied (tinnitus, dizziness, age, gender).

As it follows, the inferential static analysis was also performed, with the help of adequate tests, in order to verify:

a) The correlation between variables: Spearman Correlation test aiming to verify the level of relationship between pairs of variables of interest, such as THI x Dizziness, Gender $\mathrm{x}$ Dizziness.

b) Comparison between pitch and loudness values to each group, with or without dizziness: parametric test $t$ of Student to independent samples of interval variables of normal distribution; or its nonparametric correspondent when needed.

c) The differences were considered meaningful when $\mathrm{p} \cdot 0,05$ was presented. The static analysis was performed through the Software Statistical Package for Social Sciences (SPSS), version 20.0 .

\section{Results}

Out of the 126 evaluated individuals (64.3\%) are female and 45 (35.7\%) male, with average age of 49,33 years. All of them presented tinnitus, whom $71(56,3 \%)$ also complain about dizziness, whereas $55(43,7 \%)$ don't. Based on this, it was noticed that most part of the studied population stated complaints regarding tinnitus associated dizziness, also the static analysis shows that the variable gender has great interference in the presence or absence of dizziness. Regarding the THI questionnaire, the patients with dizziness presented bigger results for the light level, however patients without dizziness, presented bigger results to the quick level. As for tinnitus characteristics, both groups presented average pitch around $4.000 \mathrm{~Hz}$, with no meaningful statistical difference between them. On the other hand, loudness average of the tinnitus was 22 dBNS for the dizziness group and 26 dBNS for the individuals without dizziness, no meaningful difference between the groups was found (Tables 1-3).

Table 1: Descriptive and inferential statistics of the variables gender and dizziness.

\begin{tabular}{|c|c|c|c|}
\hline Gender & With Dizziness & $\begin{array}{c}\text { Without } \\
\text { Dizziness }\end{array}$ & p value \\
\hline Female & $41,3 \%(52)$ & $23 \%(29)$ & $0,017^{*}$ \\
\hline Male & $15,1 \%(19)$ & $20,6 \%(26)$ & \\
\hline
\end{tabular}

Meaningful difference $(\mathrm{p}<0,05)$ according to Spearman's correlation test. 
Table 2: Descriptive and inferential statistics of tinnitus disturbance and dizziness.

\begin{tabular}{|c|c|c|c|c|c|c|}
\hline \multirow{2}{*}{ Groups } & \multicolumn{5}{|c|}{ Values THI (\%) } \\
\cline { 2 - 7 } & Quick & Light & Moderate & Severe & Catastrophic & p value \\
\hline With Dizziness & $8,7(11)$ & $18,3(23)$ & $7,1(9)$ & $12,7(16)$ & $9,5(12)$ & 0,71 \\
\hline Without Dizziness & $14,3(18)$ & $8,7(11)$ & $10,3(13)$ & $5,6(7)$ & $4,8(6)$ & \\
\hline
\end{tabular}

Meaningful difference $(\mathrm{p}<0,05)$ according to Spearman's correlation test.

Table 3: Averages of pitch and loudness of the tinnitus.

\begin{tabular}{|c|c|c|}
\hline \multirow{2}{*}{ Groups } & \multicolumn{2}{|c|}{ Values } \\
\cline { 2 - 3 } & Average Pitch & 22,77 \\
\hline With Dizziness & 4110,91 & 26,4 \\
\hline Without Dizziness & 4922,72 & 0,234 \\
\hline$p$ value & 0,105 & \\
\hline
\end{tabular}

Meaningful differences when $\mathrm{p}<0,05$ according to the $\mathrm{t}$ Student test.

\section{Discussion}

Both tinnitus and dizziness are otoneurological complaints often presented at the practice clinic. Patients that exhibit tinnitus might also show signs of alteration and/or vestibular complaints. In this research, was observed that most of the population filed dizziness complaints. This data corroborates with literature, seeing it as complaint usually reported alongside tinnitus $[20,21]$. Data from table one shows that more women (41.3\%) presented complaints about dizziness associated with tinnitus and there was a meaningful correlation ( $p=0,017)$, in accordance with what literature shows [24-27]. It is believed that the higher occurrence among females might be due to factors such as: variation of the hormonal cycle, higher occurrence of migraines and the fact that women are more likely to seek medical attention $[27,28]$. Therefore, the variable gender directly affects the presence or not of dizziness. The disturbance caused by the tinnitus may vary greatly, and there are factors that appear to be associated with a higher level of disturbance, such as stress, psychiatric disorders and gender $[29,30]$. The THI analysis, expressed in table 2 , showed that most of the patients with dizziness presented light level 18,3\%, whereas, patients without dizziness presented the quick level 14,3\%. Therefore, the data is compatible with the ones presented by the studies of Xavier [31] and Lim et al. [32] that highlight the light and quick level as the most common among the samples of tinnitus. It can also be observed in the present study that, despite the variables THI and Dizziness lack of meaningfulness $(p=0,71)$, patients with dizziness complaints seem to exhibit a higher level of disturbance regarding tinnitus than the ones without complaint. In regards to the characteristics of the tinnitus evaluated by acuphenometry (Table 3), when compared to frequency values, both groups showed an average pitch around $4.000 \mathrm{~Hz}$, in other words, the two groups, regardless dizziness complaints, exhibited the pitch of the tinnitus in acute frequencies, with no meaningful statistical difference between them $(p=0,105)$. These findings are in accordance with the studies of Urnad and Tochetto [33] and Suzuki and collaborators [34] which also verifies values referring to the tinnitus's pitch in acute frequencies.
This is strongly related with the fact that most patients with tinnitus present hearing loss in these frequencies. The researchers state that there is a connection between the tinnitus's pitch and the region of the frequency of the maximum hearing loss. Taking intensity into consideration, the average loudness of the tinnitus was 22 dBNS for the group with dizziness and 26 dBNS for individuals without it, no verified meaningfulness between the two groups $(p=0,234)$. This data goes against the values of the studies of Buzo and Carvallo [35] and Tugumia and collaborators [36] that verified loudness varying from 5 to $15 \mathrm{dBNS}$, this way, our population shows a bigger sensation of intensity to the tinnitus. Meaningful results regarding the relation between tinnitus and dizziness were not observed in this sample. Probably, the dizziness complaints of these patients are not related to the vestibular system and, consequently, to the tinnitus. Understanding that the auditory and vestibular systems are intimately related, it becomes necessary that the patient with dizziness complaints be directed to and otoneurological evaluation, in order to investigate if the source of dizziness is vestibular, what could possibly strengthen the relationship between the tinnitus and the dizziness. Afterwards, it is possible to seek treatment in order to improve both conditions, simultaneously, as it is for vestibular rehabilitation which is used as a therapeutic process for dizziness when associated with tinnitus, possibly decreasing the level of disturbance caused by the tinnitus, as in accordance with the study presented by Zeigelboim and collaborators [37].

\section{Conclusion}

Based on the results found in the researched sample:

a) Most of the patients with tinnitus presented dizziness [38].

b) The variable female gender showed meaningfulness regarding the presence of dizziness.

c) An average pitch of around $4.000 \mathrm{~Hz}$ was found for individuals with and without dizziness. 
d) Average loudness was 22 dBNS for individuals with dizziness and $26 \mathrm{dBNS}$ for individuals without.

e) The level of disturbance of the tinnitus showed no meaningfulness/relationship with dizziness.

\section{References}

1. Zanardini FH, Zeigelboim BS, Jurkiewicz AL, Marques JM, Bassetto JM (2006) Vestibular rehabilitation in elderly patients with dizziness. Pró Fono Revista de Atualização Científica 19(2): 177-184.

2. Jurkiewicz AL, Zeigelboim BS, Mangabeira Albernaz PL (2002) Vestibular alterations in infectious processes of the central nervous system. Journal disturbances of communication 14(1): 27-48.

3. (1995) American Academy of Otolaryngology- Head and Neck Foundation, Inc. Otolaryngol Head Neck Surg 113(3): 181-185.

4. Neuhauser HK, Von Brevern M, Radtke A, Lezius F, Feldmann M, et al. (2005) Epidemiology of vestibular vertigo: a neurotologic survey of the general population. Neurology 65(6): 898-904.

5. Bittar RSM, Oiticica J, Bottino MA, Ganança FF, Dimitrov R (2013) Population epidemiological study on the prevalence of dizziness in the city of São Paulo. Braz J Otorhinolaryngol 79(6): 688-698.

6. Formigoni LG, Medeiros IRT, Santoro PP, Bittar RSM, Bottino MA (1999) Diagnostic approach of vertigo in children. Brazilian Journal of otorhinolaryngology 65(1): 78-82.

7. Medeiros IR, Bittar RS, Pedalini ME, Lorenzi MC, Formigoni LG, et al. (2005) Vestibular rehabilitation therapy in children. Otol Neurotol 26(4): 699-703.

8. Moulin T, Sablot D, Vidry E Belahsen F, Berger E, Lemounaud P, et al. (2003) Impact of emergency room neurologists on patient management and outcome. Eur Neurol 50 (4):207-214.

9. Pessoa KS (1999) Avaliação Otoneurológica, CEFAC, Rio de janeiro.

10. Staab JP (2006) Chronic dizziness: the interface between psychiatry and neuro-otology. Curr Opin Neurol 19: 41-48.

11. Yardley L (2000) Overview of psychologic effects of chronic dizziness and balance disorders. Otolaryngol Clin North Am 33(3): 603-616.

12. Knobel K, Almeida K (2001) Profile of patients in therapy for tinnitus habituation. Brasília: Rev. Fonoaudiologia Brazil.

13. Mota PHM, Franco ES, Pinto ESM, Arieta AM (2002) Study of equilibrium in elderly by electronystagmography. Acta AWHO 21(3-4): 1-12.

14. Bento RF, Miniti A, Marone SAM (1998) Diseases of the inner ear. Treaty of Otology. São Paulo: Edusp, pp. 322-331.

15. Sanchez TG, Mak MP, Pedalini MEB (2005) Study on individuals with tinnitus and normal hearing. Arq Int Otorrinolaringol 9(3): 220-227.

16. Oiticica J, Bittar RS (2015) Tinnitus prevalence in the city of São Paulo. Braz J Otorhinolaryngol 81:167-176.

17. Schleuning A (1998) Medical aspects of tinnitus. In: VERNON J, (Eds.), Tinnitus: treatment and relief. Allyn and Bacon, Boston, USA.

18. Bertet S, Baskind A, Londero A, Bonfils L, Viaud Delmon I, Warusfel 0 (2013) Design and evaluation of tinnitus synthesis methods: From spectral to spatial matching. Otolaryngology - Head and Neck medicine and Surgery 34: 121-132.

19. Cima RFF, Crombez G, Vlaeyen JWS (2011) Catastrophizing and Fear of Tinnitus Predict Quality of Life in Patients with Chronic Tinnitus. Ear \& Hearing 32(5): 634-641.
20. Moreira DF, Bohlsen YA, Momensohn Santos TM, Cherubini AA (2006) Study of the Handicap Caused by Dizziness in Patients Associated or Not with Tinnitus Complaint. Arq Int Otorrinolaringol / Intl Arch Otorhinolaryngol 10(4): 270-277.

21. Almeida LD, Mitre EI,Lemos L,Simões ECC(2005)Electronystagmography in individuals with tinnitus and normal audiology evaluation. Revista CEFAC 7(3): 382-387

22. Cohen H (2000) Vestibular rehabilitation improves daily life function. Am J Occup Ther 48(10): 919-925.

23. Azevedo AA, Oliveira PM, Siqueira AG, Figueiredo RR (2007) A critical analysis of tinnitus measuring methods. Brazilian Journal of otorhinolaryngology 73(3): 418-423.

24. Charles J, Fahridin S, Britt H (2008) Vertiginous syndrome. Aust Fam Physician 37(5): 299.

25. Kroenke K, Price RK (1993) Symptoms in the community. Prevalence, classification, and psychiatric comorbidity. Arch Intern Med 153(21): 2474-2480.

26. Neuhauser HK (2007) Epidemiology of vertigo. Curr Opin Neurol 20(1): 40-46.

27. Yardley L, Owen N, Nazareth I, Luxon L (1998) Prevalence and presentation of dizziness in a general practice community sample of working age people. Br J Gen Pract 48(429): 1131-1135.

28. Bittar RSM (1997) Hormone Labyrinth: Hormone Steroids, Estrogen and Progesterone. Int Arch Otorhinolaryngol 1(4): 32.

29. Langguth B, Kleinjung T, Fischer B, Hajak G, Eichhammer P, et al. (2007) Tinnitus severity, depression and the big five personality traits. Prog Brain Res 166: 221-227.

30. Seydel C, Haupt H, Szczepek AJ, Klapp BF, Mazurek B (2010) Long-term improvement in tinnitus after modified tinnitus retraining therapy enhanced by a variety of psychological approaches. Audiol Neurootol 15: 69-80.

31. Xavier GO (2011) Impact of tinnitus on the quality of life of socially active elderly. Porto Alegre, dez.

32. Lim JJBH, LU PK, Koh DS, Eng SP (2010) Impact of tinnitus as measured by the Tinnitus Handicap Inventory among tinnitus sufferers in Singapore. Singapore Med J 51(7): 551-557.

33. Urnad D, Tochetto TM (2011) Characteristics of tinnitus and hyperacusis in normal hearing individuals. Intl. Arch. Otorhinolaryngol 15(4): 468474 .

34. Suzuki FAB, Suzuki FA, Fernando Kaoru Yonamine FKY, Onishi ETO, Oliveira Penido NO (2016) Effectiveness of sound therapy in patients with tinnitus resistant to previous treatments: importance of adjustments. Braz J Otorhinolaryngol 82: 297-303.

35. Buzo BC, Carvallo RMM (2014) Psychoacoustic analyses of cochlear mechanisms in tinnitus patients with normal auditory thresholds. Int J Audiol 53(1): 40-47.

36. Tugumia D, Samelli AG, Carla Gentile Matas CG, Magliaro FCL, Rabelo CM (2016) Auditory training program in subjects with tinnitus. CoDAS 28(1): 27-33.

37. Zeigelboim BS, Rosa MRD, Klagenber KF, Jurkiewicz AL (2008) Vestibular rehabilitation in the treatment of dizziness and tinnitus. Rev Soc Bras Fonoaudiol 13(3).

38. Figueiredo RR, Azevedo AA, Oliveira PM (2009) Correlation analysis of the visual-analogue scale and the Tinnitus Handicap Inventory in tinnitus patients. Brazilian journal of otorhinolaryngology. 75(1). 
This work is licensed under Creative Commons Attribution 4.0 License

To Submit Your Article Click Here: Submit Article

DOI: $10.32474 /$ SJO.2019.02.000128

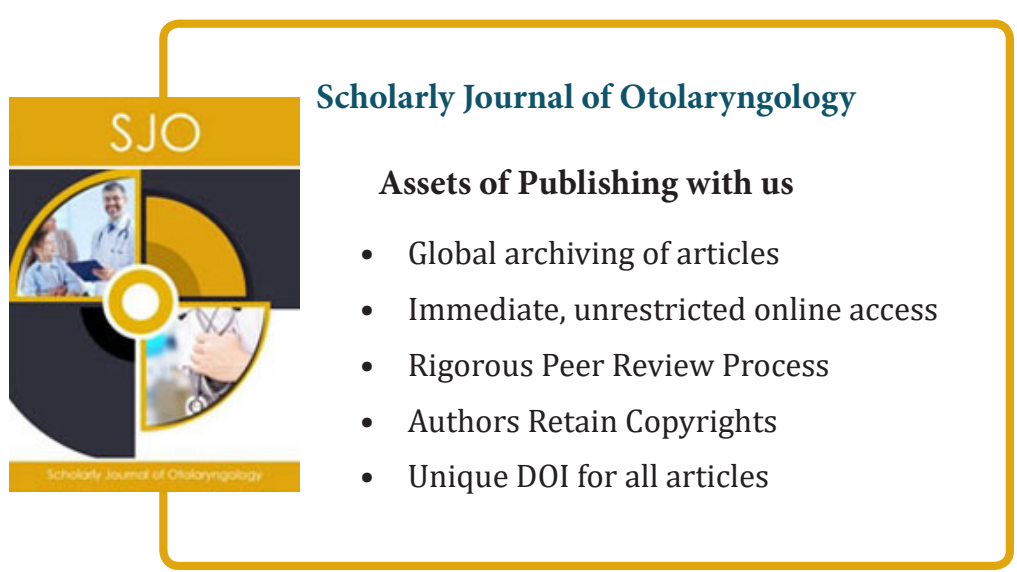

